\title{
OPEN CRISPR-based transcriptional activation tool for silent genes in filamentous fungi
}

\author{
László Mózsik ${ }^{1,6}$, Mirthe Hoekzema ${ }^{1,5,6}$, Niels A. W. de Kok ${ }^{1}$, Roel A. L. Bovenberg ${ }^{2,3}$, \\ Yvonne Nygård ${ }^{1,2,4}$ \& Arnold J. M. Driessen ${ }^{1 凶}$
}

Filamentous fungi are historically known to be a rich reservoir of bioactive compounds that are applied in a myriad of fields ranging from crop protection to medicine. The surge of genomic data available shows that fungi remain an excellent source for new pharmaceuticals. However, most of the responsible biosynthetic gene clusters are transcriptionally silent under laboratory growth conditions. Therefore, generic strategies for activation of these clusters are required. Here, we present a genome-editing-free, transcriptional regulation tool for filamentous fungi, based on the CRISPR activation (CRISPRa) methodology. Herein, a nuclease-defective mutant of Cas9 (dCas9) was fused to a highly active tripartite activator VP64-p65-Rta (VPR) to allow for sgRNA directed targeted gene regulation. dCas9-VPR was introduced, together with an easy to use sgRNA "plug-and-play" module, into a non-integrative AMA1-vector, which is compatible with several filamentous fungal species. To demonstrate its potential, this vector was used to transcriptionally activate a fluorescent reporter gene under the control of the penDE core promoter in Penicillium rubens. Subsequently, we activated the transcriptionally silent, native $P$. rubens macrophorin biosynthetic gene cluster by targeting dCas9-VPR to the promoter region of the transcription factor macR. This resulted in the production of antimicrobial macrophorins. This CRISPRa technology can be used for the rapid and convenient activation of silent fungal biosynthetic gene clusters, and thereby aid in the identification of novel compounds such as antimicrobials.

$\begin{array}{ll}\text { Abbreviations } \\ \text { BGC } & \text { Biosynthetic gene cluster } \\ \text { CRISPRa } & \text { CRISPR activation } \\ \text { SM } & \text { Secondary metabolite } \\ \text { cDNA } & \text { Complementary DNA } \\ \text { sgRNA } & \text { Chimeric single guide RNA } \\ \text { RNPs } & \text { Ribonucleoprotein complexes } \\ \text { NLS } & \text { Nuclear localization signal } \\ \text { CP } & \text { Core promoter } \\ \text { TSS } & \text { Transcription start site }\end{array}$

Fungi are amongst the most proliferous producers of secondary metabolites (SMs). These molecules, while not intrinsically required for survival, provide a biological advantage to their host ${ }^{1}$. Many fungal SMs are beneficial to humankind and have a wide range of applications in human and animal healthcare (e.g. as antibiotics or immunosuppressants), food, agricultural and industrial sectors ${ }^{2,3}$. On the other hand, SMs can be toxic and some SMs contribute to the pathogenicity of fungi while others contaminate food and crops $^{4}$. Genes involved in secondary metabolism are often arranged in clusters, so-called biosynthetic gene clusters (BGCs), and these

\footnotetext{
${ }^{1}$ Molecular Microbiology, Groningen Biomolecular Sciences and Biotechnology Institute, University of Groningen, Nijenborgh 7, 9747 AG Groningen, The Netherlands. ${ }^{2}$ DSM Biotechnology Center, Alexander Fleminglaan 1, 2613 AX Delft, The Netherlands. ${ }^{3}$ Synthetic Biology and Cell Engineering, Groningen Biomolecular Sciences and Biotechnology Institute, University of Groningen, Nijenborgh 7, 9747 AG Groningen, The Netherlands. ${ }^{4}$ Division of Industrial Biotechnology, Department of Biology and Biological Engineering, Chalmers University of Technology, Kemivägen 10, 41296 Gothenburg, Sweden. ${ }^{5}$ Division of Forest Pathology, Department of Forest Mycology and Plant Pathology, Swedish University of Agricultural Sciences, Almas allé 5, 75651 Uppsala, Sweden. ${ }^{6}$ These authors contributed equally: László Mózsik and Mirthe Hoekzema. ${ }^{\circledR}$ email: a.j.m.driessen@rug.nl
} 
are typically regulated by pathway-specific transcription factors. As more fungal genomes, and bioinformatics tools and databases (e.g. fungal antiSMASH${ }^{5}, \mathrm{MIBiG}^{6}$ ) have become available for the prediction, annotation and prioritization of fungal BGCs, it has become clear that filamentous fungi have an even larger biosynthetic potential than previously anticipated.

Most of the BGCs are transcriptionally silent under laboratory growth conditions, therefore products of these clusters remain elusive ${ }^{7}$. Various methodologies have been developed for the activation of silent BGCs, including manipulation of both BGC specific as well as global transcriptional regulators, promoter-exchange, and heterologous expression in suitable host systems [reviewed in ${ }^{8}$ ]. Marker-free genome editing remains challenging, and with only a limited number of fungal selection markers available, extensive genome manipulations is a laborious task.

The bacterial CRISPR/Cas systems have emerged as versatile biotechnological tools ${ }^{9,10}$, and next to genome editing it can provide a promising alternative approach for transcriptional activation in fungi. CRISPR/Cas systems consist of only two components; a Cas nuclease and a programmable guide RNA. In case of the popular Cas9 system from Streptococcus pyogenes the protein can be guided to a genomic locus in a sequence-specific manner, using a single guide RNA (sgRNA) which consist of a short targeting crRNA sequence and the scaffold tracrRNA sequence. Methods for Cas9-based genome editing have been established in various filamentous fungal species [Reviewed ${ }^{11,12}$ ], including the industrially relevant fungi Penicillium rubens ${ }^{13,14}$ (formerly identified as P. chrysogenum ${ }^{15}$ ). Cas 9 and sgRNA delivery strategies include vector-based expression or genomic integration of transcriptional units encoding Cas 9 and sgRNA. Alternatively, only Cas 9 is expressed and the sgRNA is provided by a transformation of in vitro transcribed RNA, or both Cas 9 and sgRNA are provided as pre-assembled ribonucleoprotein complexes (RNPs). The CRISPR/Cas9 genome editing tools established in filamentous fungi can edit the genome at a single as well as at multiple locations, and have effectively been applied in industrial fungi to improve compound production ${ }^{11,12}$.

Beyond genome editing, CRISPR/Cas can be used as a platform for RNA guided DNA-protein interactions, and thereby deliver various effector domains to a specific genomic location. By introducing point mutations into the two nucleolytic domains, nuclease deficient versions of Cas9, called dead Cas9 (dCas9), were created ${ }^{16}$. Because dCas 9 binds in a sequence-specific manner, but does not cleave DNA, it can be used for transcriptional regulation $^{17,18}$, epigenome editing ${ }^{19,20}$, visualization of specific genomic loci ${ }^{21}$ and base editing ${ }^{22}$ in various eukaryotic species. For CRISPR/Cas mediated transcriptional activation (CRISPRa) several activating effector domains have been fused to dCas9 [reviewed in ${ }^{23,24}$ ]. The often-used VPR system consists of a three-component complex, four copies of the herpes simplex VP16 transactivation domain, the transactivation domain of nuclear factor kappa B, and Epstein-Barr virus R transactivator, VP64-p65-Rta, respectively ${ }^{16}$. dCas-VPR fusions have been successfully employed for upregulation of reporter and/or endogenous genes in mammalian cells ${ }^{16}$, in diploid ${ }^{25}$ and polyploid ${ }^{26}$ Saccharomyces cerevisiae, Yarrowia lipolytica ${ }^{27}$, Candida albicans ${ }^{28}$, and most recently also in the filamentous fungus Aspergillus nidulans ${ }^{29}$.

Here we report on the implementation of a dCas9-VPR-based, genome editing free system for transcriptional activation system in the filamentous fungus Penicillium rubens. We successfully utilized the CRISPRa tool to activate the cryptic macrophorin BGC, resulting in production of compounds with antimicrobial activity.

\section{Results}

Construction of a fungal CRISPRa tool. CRISPR/Cas mediated gene expression activation (CRISPRa) requires a catalytically dead CRISPR-associated protein (dCas) fused to an activation domain, as well as a sgRNA to guide it to the desired locus. Here, the widely utilized fusion of dCas9 from Streptococcus pyogenes to the tripartite activator, VP64-p65-Rta (VPR ${ }^{16}$ was selected for activation. For easy implementation of CRISPRa in a broad range of filamentous fungi, we constructed an AMA1-based vector for expression of the NLS tagged dSpCas9-VPR under the 40S ribosomal protein S8 promoter (p40S) (Fig. 1a). The AMA1 sequence -originally isolated from A. nidulans- allows for autonomous vector replication in several filamentous fungal species ${ }^{30,31}$, and is often employed for Cas9 and sgRNA expression in gene-editing approaches in these organisms ${ }^{11,13,32,33}$. The AMA1 vector was also used to supply the sgRNA, establishing CRISPRa after a single transformation. The sgRNA was expressed from the constitutive $g p d A$ promoter and flanked by hammerhead $(\mathrm{HH})$ and hepatitis delta virus (HDV) ribozymes to ensure defined ends for sgRNA processing and optimal functionality (Fig. 1a) ${ }^{34}$.

Target specificity is determined by the sgRNA, thus by exchanging the sgRNA sequence different genes can be targeted for upregulation. To enable convenient and efficient exchange of sgRNA target sequences a sgRNA "plug-and-play" module was introduced into the AMA1 shuttle vector to facilitate cloning steps in Escherichia coli (Fig. 1a,b). This vector, which is the parent to all sgRNA expressing vectors, is called pLM-AMA18.0-dCas9VPR (referred to as pAMA18.0) and also functions as a negative (non-targeting sgRNA) control. The sgRNA "plug-and-play" module works as follows; the chimeric sgRNA backbone sequence and the HDV ribozyme are already supplied on the AMA1-vector together with a lacZ gene flanked by BsaI restriction sites. The 20 nt spacer sequence defining the genomic target is supplied on a separate dsDNA molecule, together with the hammerhead ribozyme $(\mathrm{HH})$ which includes the necessary $6 \mathrm{bp}$ inverted repeat of the 5 '-end of the spacer to complete the $\mathrm{HH}$ cleavage site. This dsDNA molecule can simply be created by PCR using two overlapping oligonucleotides (Fig. 1b) or alternatively ordered as chemically synthetized dsDNA. The fragment can then be inserted into pAMA18.0 using the Golden Gate cloning and the BsaI restriction sites ${ }^{35}$. As this removes the lac $Z$ gene, positive bacterial clones can easily be detected with blue-white screening. After positive sequence verification and vector extraction, the created CRISPRa vector can be introduced into the filamentous fungi of choice (Fig. 1b).

Proof of principle: activating penDE-CP_DsRed. In order to test if expression of dCas9-VPR and the sgRNA from the CRISPRa vector could activate transcription of a silent gene, we targeted dCas9-VPR to the 

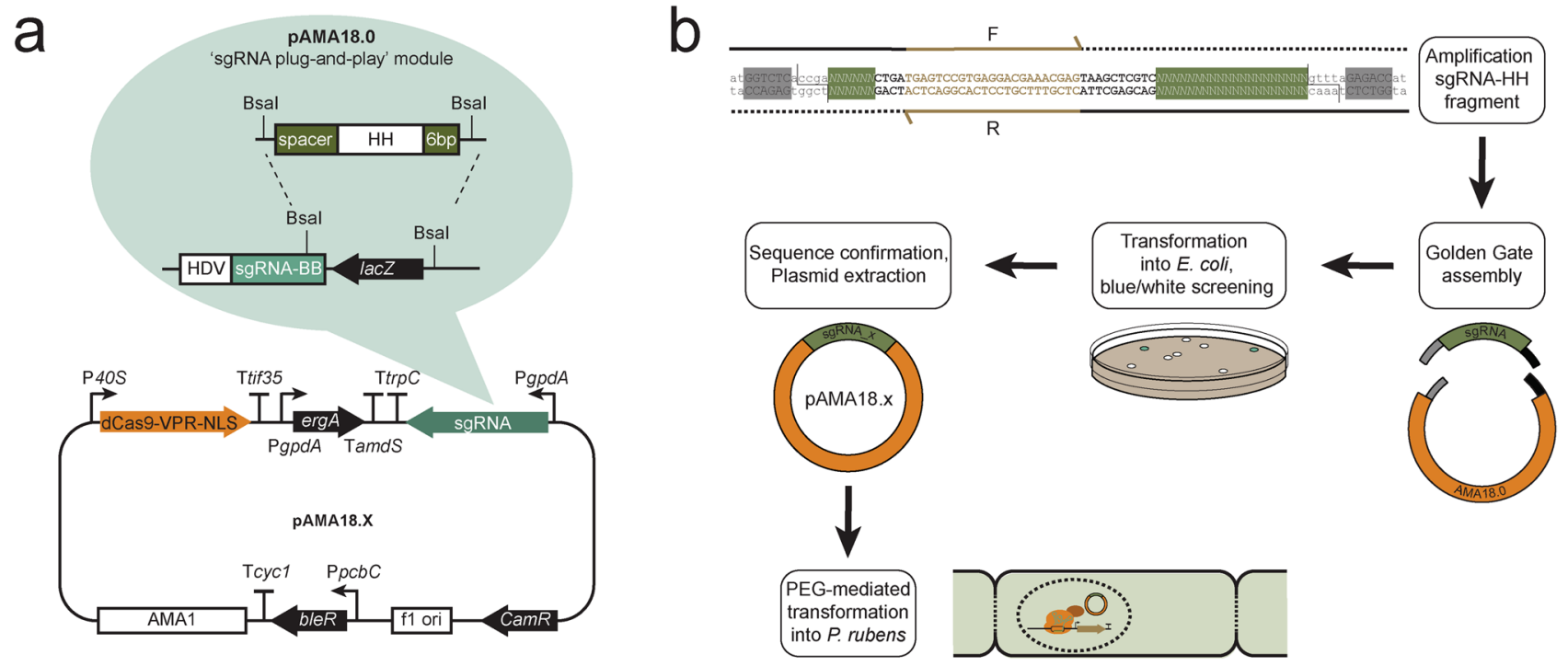

C

Negative control - no sgRNA expression

\section{CRISPRa - targeting gene of interest}
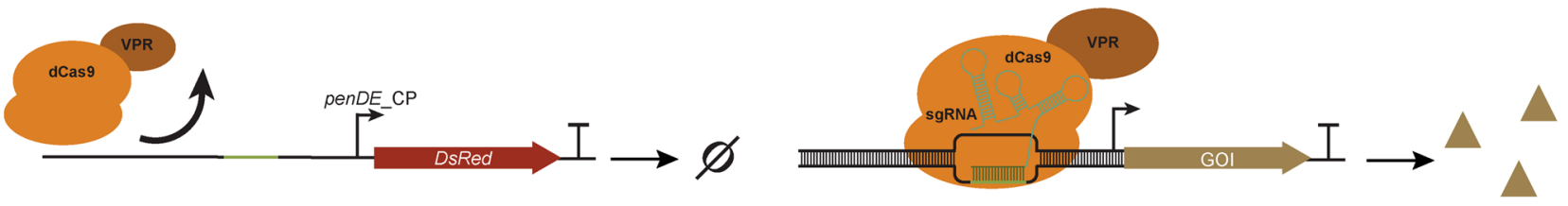

Proof of principle - activating PenDE_CP:DsRed
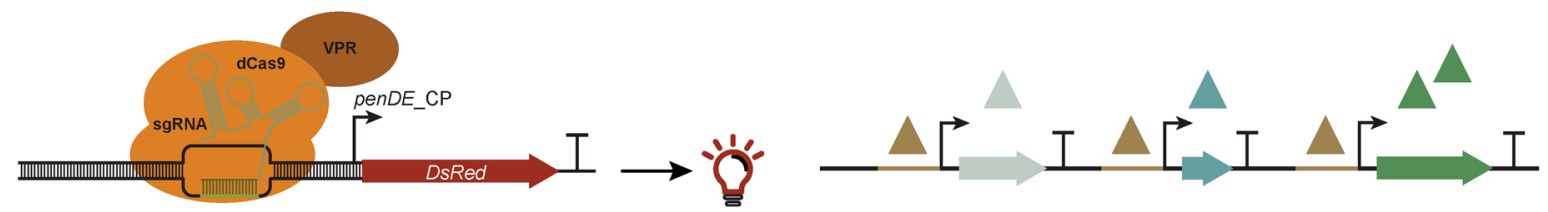

Figure 1. Overview of the programmable CRISPR/Cas-based transcriptional activation system implemented in P. rubens. (a) Schematic representation of the pAMA18.X-vector encoding the components of the CRISPR/Cas activation system, namely the dCas9-VPR and the ribozyme self-cleaved sgRNA. pAMA18.0 is the parent vector of all sgRNA containing vectors and contains the sgRNA "plug-and-play" module which is highlighted. (b) Diagram depicting the cloning strategy for insertion of the PCR amplified sgRNA into pAMA18.0. (c) CRISPRa proof of principle. In the control strain carrying pAMA18.0 no sgRNAs are transcribed, so while dCas9-VPR is present it is not targeted to a specific locus and no transcriptional activation occurs. Correct targeting of the dCas9-VPR complex to the silent penDE-CP is leading to DsRed fluorescent protein expression and hence increased fluorescence. In the same fashion when dCas9-VPR is targeted to a promoter driving a gene of interest, this results in product formation. When the targeted promoter drives a transcriptional regulator this can result in activation/repression of multiple other genes, including entire BGCs.

penDE core promoter (penDE-CP). The $200 \mathrm{bp}$ long penDE-CP has previously been shown to be functional, but insufficient to drive expression on its $\mathrm{Own}^{36}$. For easy visualization of CRISPR based transcriptional activation, the penDE-CP was set to drive DsRed-T1-SKL, a red fluorescent reporter gene with peroxisomal targeting signal (Fig. 1c). The penDE-CP_DsRed reporter unit was integrated into the penicillin-locus of the P. rubens DS68530 ( $\triangle$ penicillin-BGC), utilizing CRISPR/Cas9 ribonucleoprotein (RNP) facilitated co-transformation ${ }^{13,14}$ (Supplementary Fig. S1a).

Different pAMA18.0 derived vectors (pAMA18.a-f) expressing sgRNAs targeting loci +1 to -118 bp relative to the transcription start site (TSS) of the penDE-CP (Fig. 2a, Supplementary Fig. S2a, Supplementary Table S1) were transformed into $P$. rubens DS68530_penDE-CP_DsRed and strains were analyzed using fluorescence microscopy (Fig. 2b). Increased fluorescence intensity was seen in strains transcribing penDE-sgRNA_c, d, and _e but not in strains transcribing penDE-sgRNA_a, _b and_f. The DS68530_penDE-CP_DsRed strain carrying the pAMA18.0 negative control vector which did not express any sgRNA, showed only a minimal amount of fluorescence. DsRed expression was also evaluated using qPCR, showing the most efficient activation for penDE-sgRNA_c (Fig. 2c). These results confirm that activation of DsRed expression was CRISPRa dependent.

To assess the performance of the different sgRNA target sequences, the BioLector microbioreactor system was used with online monitoring of scattered light (biomass) and red fluorescence intensity (Fig. 2d, Supplementary 

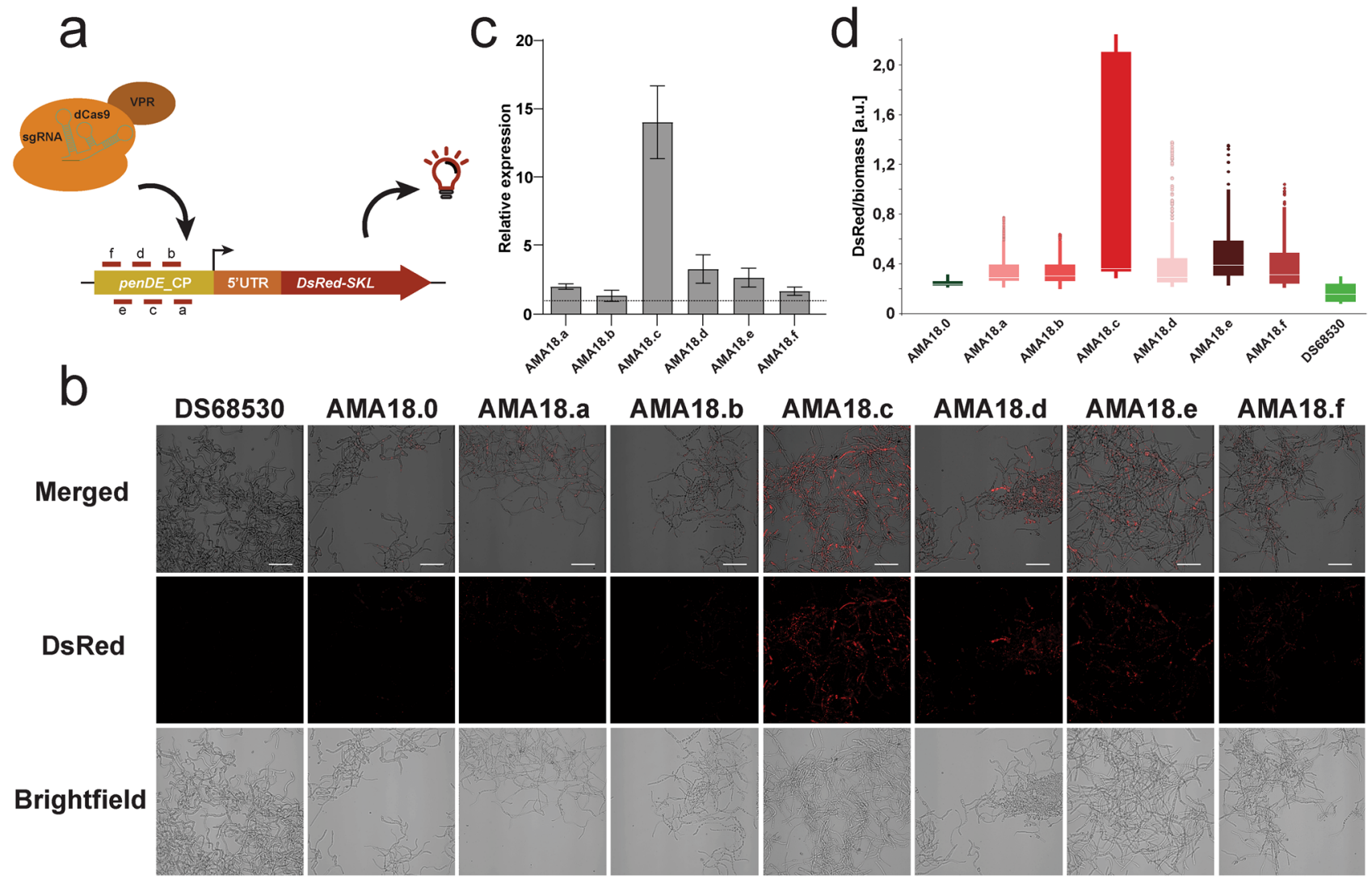

Figure 2. CRISPRa (dCas9-VPR) based activation of penDE-CP_DsRed. (a) Schematic representation of the penDE-CP upstream DsRed. The transcription start site (TSS) is indicated as a black arrow, short lines with letters indicate targeting sites of the sgRNAs. (b) Confocal fluorescence microscopy imaging of DsRed targeting CRISPRa strains and controls with no-sgRNA (AMA18.0) and without the penDE-CP_DsRed transcription unit (DS68530). Strains were grown for 5 days in liquid SMP media. Scale bars represent $50 \mu \mathrm{m}$. (c) qPCR analysis showing expression levels of DsRed in CRISPRa strains relative to strain carrying pAMA18.0 no-sgRNA negative control (dotted line) after 5 days of growth in SMP. (d) Development of DsRed/biomass over time during time window of $0-40 \mathrm{~h}$ cultivation in the BioLector system. Data were obtained from 3 separate experiments, each consisting of 2-3 biological replicates; error bars show the standard deviation.

Fig. S3). The strength of DsRed activation by different sgRNAs was determined relative to biomass to avoid variance caused by small differences in growth. During the time interval of $0-40 \mathrm{~h}$, DsRed/biomass values in CRISPRa strains were measured and compared to negative control strain carrying pAMA18.0 and the background fluorescence of the DS68530 parental strain. The pAMA18.c carrying strain showed the highest level of relative fluorescence and thus provided the most efficient activation compared to the non-sgRNA negative control (Fig. 2d). All other CRISPRa strains show activation of penDE-CP_DsRed, with weakest activation in strains carrying pAMA18.a and pAMA18.b vectors.

CRISPRa-based activation of the transcriptionally silent macrophorin gene cluster. Meroterpenoids represent a large family of natural compounds with diverse biological activities, such as the antimicrobial yanuthones found in Aspergillus niger ${ }^{37,38}$. Highly identical clusters have been found in Penicillium species ${ }^{39}$. These Penicillium BGCs contain an additional gene (macJ), which was shown in Penicillium terreste to encode a terpene cyclase responsible for cyclization of linear yanuthones leading to production of diverse macrophorin analogs ${ }^{39}$. The putative $P$. rubens macrophorin BGC consists of 11 biosynthetic genes, namely macA-J and macR as a transcriptional regulator of the cluster (Fig. 3a,b).

Sequence alignment of the provisional sequence of $P$. rubens macR (Pc16g00410) to the P. terrestre LM2 macR coding sequence (MF989995.1) shows that the P. rubens sequence is predicted to have an additional intron leading to a premature stop codon. Without this intron, the P. rubens macR mRNA should produce a full-length product, similarly to $P$. terrestre LM2 $m a c R$. To test if $m a c R$ codes for a functional protein we performed promoter replacement in P. rubens DS68530, substituting the promoter region of $m a c R$ with the promoter of the $p c b C$ (isopenicillin $\mathrm{N}$ synthase) gene (Supplementary Fig. S1b), creating strain macR:OE. The resulting increase in macR transcription (Fig. 3c) led to the activation of the cryptic BGC (Fig. 3d,e) and the production of macrophorins (Fig. 3f,g, Supplementary Table S2). We therefore conclude that $P$. rubens macR encodes for a functional transcription factor and that increased expression of $m a c R$ leads to activation of the entire associated BGC. Moreover, activation of this BGC leads to production of macrophorin-like compounds (Supplementary Table S2). 
a

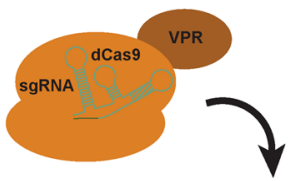

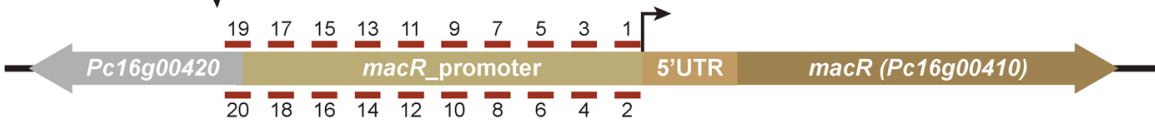

b

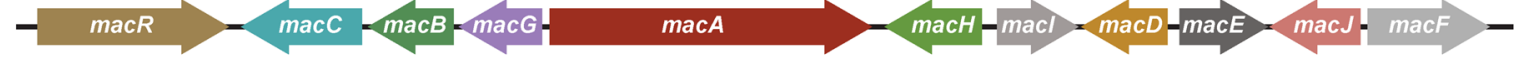

C

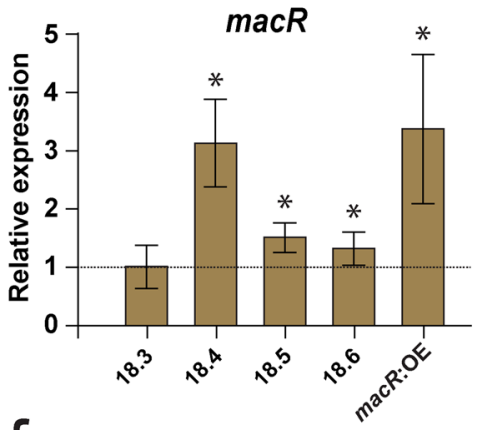

f

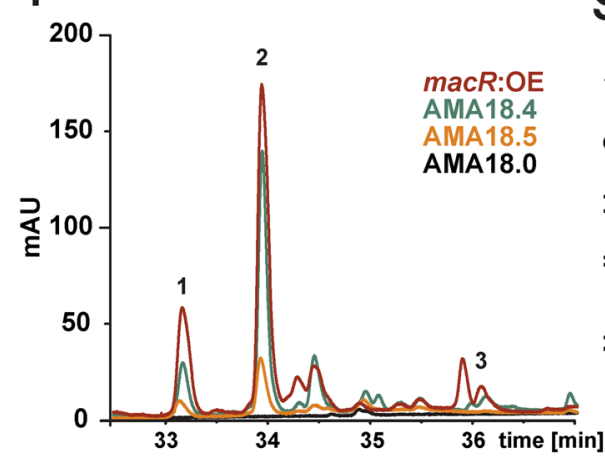

d

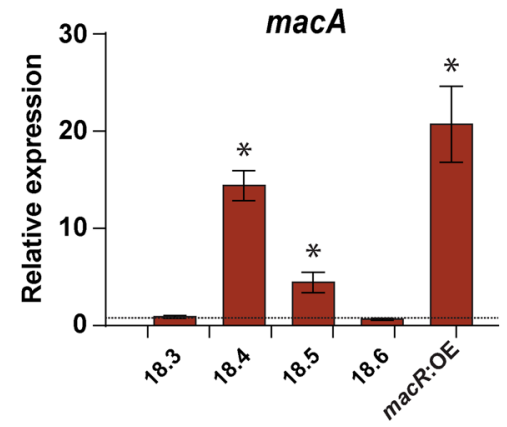

g

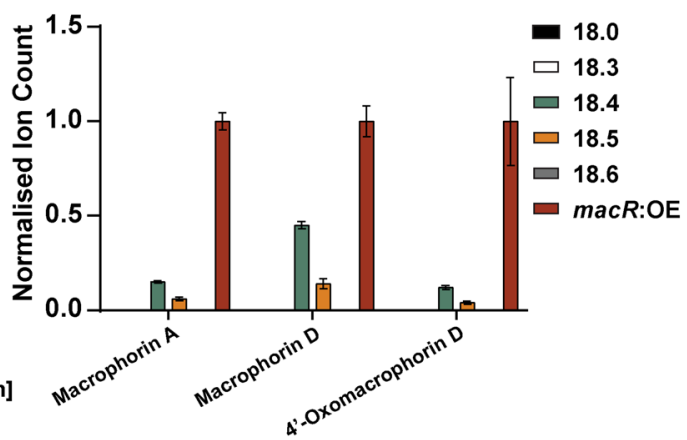

macJ
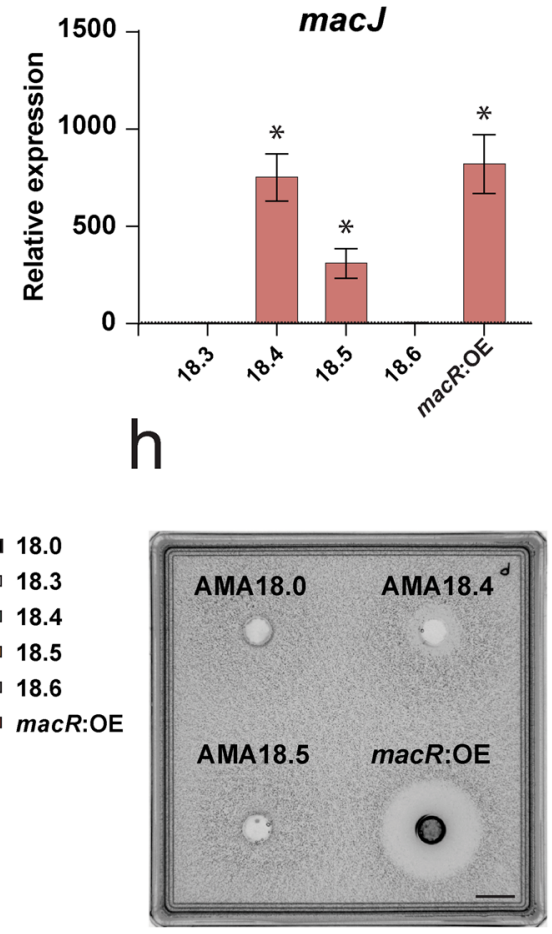

Figure 3. CRISPRa activation of the macrophorin BGC. (a) Schematic representation of the macR promoter region. The location of the putative transcription start site (TSS) is indicated as a black arrow, short lines with number indicate sgRNAs targeting sites. (b) Schematic representation of the Macrophorin BGC. qPCR analysis showing expression of $\operatorname{macR}(\mathbf{c}) \operatorname{macA}(\mathbf{d})$ and $\operatorname{macJ}(\mathbf{e})$ in the CRISPRa and macR:OE strains, relative to the strain carrying the pAMA18.0 vector (non-target control) after 5 days of growth in SMP medium. Error bars indicate the standard error of the mean of three biological replicates with two technical duplicates, and $\left(^{*}\right)$ indicates significant up-regulation (Student's-test p-value $<0.05$ ). (f) LC-MS UV-VIS chromatogram $(\lambda=700 \mathrm{~nm})$ of hyphae extracts of CRISPRa and macR:OE strains representing macrophorin A (1), macrophorin D (2) and 4'-oxomacrophorin D (3). (g) LC-MS analysis of Macrophorin related compounds in hyphae extracts of CRISPRa and macR:OE strains. Error bars indicate the standard error of the mean of three biological replicates with two technical duplicates. (h) Bioassay to detect (macrophorin related) antimicrobial activity against Micrococcus luteus in the supernatant of indicated strains grown for 5 days in liquid SMP medium. The supernatant was concentrated 10-times and $100 \mu \mathrm{l}$ was loaded in a well in top agar containing $M$. luteus at $\mathrm{OD}_{600}=0.0125$. Scale bar represents $10 \mathrm{~mm}$.

Sanger sequencing data of cDNA obtained from the macR:OE strain showed 2 introns in $P$. rubens macR $m R N A$ and no pre-mature stop codon, in line with the coding sequence of macR of $P$. terrestre (MF989995.1) and the homologous yanR (ASPNIDRAFT_44961) of A. niger. It therefore seems likely that the third intron in the provisional $P$. rubens macR sequence is wrongly predicted, and $P$. rubens is capable of producing, not only functional, but also full-length MacR. Additionally, a mutation (cDNA 2611C > T, P776S) mutation was identified in the ORF of $m a c R$. The effect of this mutation was not further investigated as $m a c R$ remained capable of transcriptional activation. The sequence of $P$. rubens DS68530 macR cDNA can be found in Supplementary Note S1. 
Since the $P$. rubens macrophorin BGC is silent under our growth conditions (Secondary Metabolite Producing [SMP] medium, $\left.25^{\circ} \mathrm{C}\right)^{40,41}$, it was selected for activation by CRISPRa. As no TSS is known for macR, 20 sgRNAs (MacR-sgRNA_1-20) were designed to target the entire $547 \mathrm{bp}$ long, native promoter (Fig. 3a, Supplementary Table S1, Supplementary Fig. S2b). The macR targeting CRISPRa strains and the macR:OE positive control, were grown on SMP-agar for 10 days after which secondary metabolites were extracted from representative agar plugs, and analyzed by LC-MS (Supplementary Table S3). As expected, no macrophorin production was observed in the strain carrying the pAMA18.0 negative control with no sgRNA insert. Strains expressing MacR-sgRNA_4 and MacR-sgRNA_5 showed production of compounds with masses corresponding to macrophorin A ( $361.24 \mathrm{~m} / \mathrm{z}$ $\left.[\mathrm{M}+\mathrm{H}]^{+}\right)$, macrophorin $\mathrm{D}\left(505.28 \mathrm{~m} / \mathrm{z}[\mathrm{M}+\mathrm{H}]^{+}\right)$and $4^{\prime}$-oxomacrophorin $\mathrm{D}\left(503.26 \mathrm{~m} / \mathrm{z}[\mathrm{M}+\mathrm{H}]^{+}\right)$(Supplementary Table S2). None of the other CRISPRa strains exhibited macrophorin production.

Fungal strains carrying vector pAMA18.3-6 and pAMA18.0 (no sgRNA control) were further investigated by qPCR (Fig. 3c-e) and metabolite profiling (Fig. 3f,g). Strains expressing MacR-sgRNA_4 and sgRNA_5 were selected as these sgRNAs showed activated macrophorin production (Supplementary Table S3). Although strains carrying MacR-sgRNA_3 and sgRNA_6 did not show macrophorin production these strains were also investigated further, as these sgRNAs target the $\operatorname{macR}$ promoter region in close proximity to the successfully activating MacR-sgRNA_4 and sgRNA_5, but on the opposite strand of the DNA. As expected, strains carrying the pAMA18.4 or pAMA18.5 CRISPRa vector showed an increase in macR expression compared to the pAMA18.0 control, further confirming CRISPRa dependent transcriptional activation (Fig. 3c). The increase in macR expression resulted in transcriptional activation of the macrophorin BGC as exemplified by increased levels of macA (polyketide synthase) (Fig. 3d) and macJ (proposed terpene cyclase ${ }^{39}$ ) mRNA (Fig. 3e), that respectively encode the first and last enzymes in the macrophorin biosynthesis pathway ${ }^{39}$.

In the strain carrying the pAMA18.4 vector, levels of transcriptional activation were comparable to those in the positive control macR:OE while strain carrying vector pAMA18.5 showed a $\sim$ threefold lower transcription compared to this control, for all genes investigated (Fig. 3c-e). No increased expression of macR, $\operatorname{macA}$ or $\mathrm{macJ}$ was observed for the strain carrying pAMA18.3. In the strain carrying vector pAMA18.6, a slight upregulation of $m a c R$ was observed but this did not result in induction of macA and macJ (Fig. 3c-e). In line with this, the strain carrying pAMA18.5 produced lower amounts of the examined macrophorin related metabolites compared to the strain with pAMA18.4 (Fig. 3f,g). However, while qPCR analysis showed similar mRNA levels between the macR:OE and pAMA18.4 strains, compound production for macrophorin A and 4'-oxomacrophorin D was lower in AMA18.4 compared to the macR:OE strain, reaching 15\% and 13\% respectively. Strain AMA18.4 reached highest production for macrophorin $\mathrm{D}$ at $\sim 38 \%$ of the ion intensity measured in macR:OE.

As the related yanuthones produced by $A$. niger display antimicrobial activity against gram positive bacteria ${ }^{42}$, we analyzed the activity of our macrophorin producing Penicillium strains against Micrococcus luteus using the agar diffusion method. The transformed parent strain P. rubens DS68530 does not contain the penicillin BGC, and consequently does not produce compounds inhibiting the growth of $M$. luteus. We observed a clearance zone around concentrated supernatant from the macR:OE strain grown for 5 days in SMP medium, and to a lesser extent also around that of the AMA18.4 strain, but not that of the control (AMA18.0) or the AMA18.5 strain (Fig. $3 \mathrm{~h}$ ). This indicates that the macrophorins produced by P. rubens are indeed bioactive against Grampositive bacteria, and CRISPRa dependent activation of the BGC is sufficient to induce antimicrobial activity.

Interestingly, we observed a dark brownish pigmentation of the hyphae of the macR:OE strain after 5 days of cultivation on R-agar and SMP-agar as well as on day 1 in SMP liquid medium. The strain carrying the CRISPRa vector pAMA18.4 displayed a milder coloration compared to the colorless hyphae of the parent strain (Fig. 4). Color formation in these $\operatorname{macR}$ over-expression strains was not investigated further.

\section{Discussion}

In this work, we report the application of dCas9-VPR based CRISPRa in the ascomycetous filamentous fungus Penicillium rubens. While Penicillium is acclaimed for its production of B-lactam antibiotics, it harbors many more BGCs of which a substantial portion remain uncharacterized ${ }^{43}$. CRISPRa systems have been established in many model organisms as an ideal technology for transcriptional regulation and could aid in activating these often silent BGCs to facilitate characterization.

In our approach dCas9-VPR and the sgRNA are episomally encoded on the same AMA1-based vector, hence a single transformation with a single vector is enough to establish CRISPR-based transcriptional activation in Penicillium, without the need for genome engineering of the host organism. Moreover, because AMA1 supports autonomous vector replication in several filamentous fungal species ${ }^{30,31}$, and as we use established fungal promoters, terminators, and ribozyme based sgRNA processing, we expect the vector to be transferable to other fungal species. The sgRNA "plug-and-play" module of our CRISPRa vector combines Golden Gate cloning approach with blue/white screening. This allows for convenient cloning of new sgRNAs into the vector, reducing experimental time. This is especially important since general criteria for successful sgRNA design are difficult to define, and empiric testing of sgRNAs for each promoter region of interest remains necessary. Due to the ease of cloning our AMA1 vector, this CRISPRa technology could potentially be implemented in connection with larger scale fungal protoplast transformations using microtiter plates ${ }^{44}$, for example in combination with deploying multiple, separate sgRNA processing vectors in one transformation ${ }^{45}$.

To assess the CRISPRa vector for activation of transcriptionally silent promoter activation, we integrated a penDE core promoter driven DsRed gene into the penicillin-locus of $P$. rubens DS68530 ( $\Delta$ penicillin-BGC). This penDE-CP was selected because it has been reported previously to be insufficient to express the fluorescent reporter on its own, instead depending on the presence of a synthetic transcription factor ${ }^{36}$. Fluorescence microscopy showed a clear increase in fluorescence with 3 out of 6 sgRNAs tested, compared to a non-sgRNA expressing control (Fig. 2b). Quantification of fluorescence using a BioLector microbioreactor showed increased 


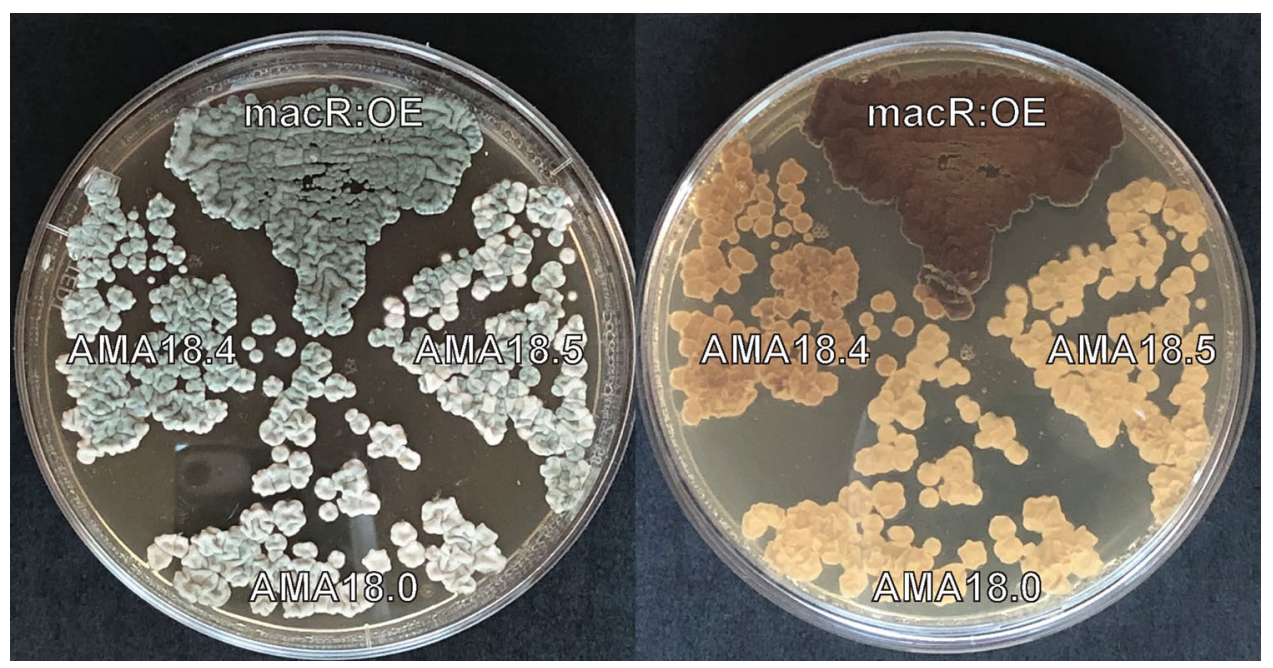

Figure 4. Dark pigmentation of hyphae due to macR overexpression in Penicillium rubens DS68530 macR:OE and in the CRISPRa pAMA18.4 vector carrying strain after 5 days of cultivation on R-agar, compared to AMA18.0 strain carrying pAMA18.0 (no sgRNA) negative control.

fluorescence for 6 out of 6 sgRNA used, showing weak activation for penDE-sgRNA_a and penDE-sgRNA_b sgRNAs, and, in line with fluorescence microscopy results, penDE-sgRNA_c standing out as the most efficient activator (Fig. 2d). The discrepancy between fluorescence microscopy and the BioLector results could possibly be explained by a higher sensitivity of the BioLector, different cultivation method and time points (day 5 of shake-flask cultures for microscopy, average fluorescent during the first $40 \mathrm{~h}$ for the BioLector cultivations).

In A. niger, Roux and co-workers observed that dCas9-VPR mediated activation of a mCherry fluorescent reporter fused to the transcriptionally silent Parastagonospora nodorum elcA promoter was stronger with sgRNAs targeting closer to the start codon, in a window of 162-342 bp upstream of the ATG $^{29}$. We target a region 106-170 bp upstream the start codon ATG (32-96 bp upstream the TSS) and observe the highest activation with penDE-sgRNA_c targeting 129 bp upstream the ATG, and the least with penDE-sgRNA_a and _b (not detectable by microcopy) targeting closer to the start codon. We thus do not see the same trend-stronger CRISPRa for sgRNAs targeting closer to the start codon-however we already target a window closer to the ATG compared to Roux et al. ${ }^{29}$. This exemplifies that it remains difficult to define an optimal targeting conditions, and ideally several sgRNAs should be tested when establishing CRISPRa for a new promoter. In line with what previously was reported for S. cerevisiae, we did not observe an effect on CRISPRa efficiency when targeting the plus or minus strand ${ }^{46}$.

To show our CRISPRa system can upregulate an entire silent BGC in P. rubens and induce metabolite production, we targeted the macR transcription factor of the endogenous macrophorin biosynthesis cluster. Macrophorins are a member of the meroterpenoids, a family of natural compounds which also include, for example, the antimicrobial yanuthones produced by $A$. niger ${ }^{37,38}$. Homologous macrophorin BGC have been identified in Penicillium species, and P. terreste has been shown to produce macrophorins, through the cyclization of yanuthones ${ }^{39}$. Out of the $20 \mathrm{sgRNAs}$ tested, two resulted in transcriptional activation of the Macrophorin BGC (through the activation of transcriptional factor $m a c R$ ) (Fig. 3c,d) and secondary metabolite production (Fig. 3f,g). Although it is impossible to distinguish macrophorins and yanuthones with the method used as they have the same molecular formula, activation of the macJ terpene cyclase should lead to cyclic macrophorins ${ }^{39}$. Additionally we could show that the supernatant of the CRISPRa activated strain grown five days in SMP media exhibited antimicrobial activity against the Gram-positive bacterium M. luteus (Fig. 3h). This clearly shows that our dCas9-VPR vector is capable of awakening silent BGCs in Penicillium and that the method can aid in product identification and characterization. It should be noted that exchanging the native $m a c R$ promoter with the $p c b C$ promoter resulted in higher compound production (Fig. $3 \mathrm{~g}$ ). It might therefore be beneficial to perform promoter exchange for high level production of interesting compounds identified using the CRISPRa technology. A possible explanation for why a larger proportion of the sgRNAs targeting penDE-CP (6/6) lead to transcriptional activation compared to $\operatorname{macR}(2 / 20)$ may be that the CP is free from most of its native regulatory elements, reducing chances of interference with the binding of the dCas9-VPR regulator. A limiting factor for this way of BGC activation is the need to identify a positive regulator for the cluster, which might not always be known. However, bioinformatics tools like antiSMASH ${ }^{47}$ or CASSIS ${ }^{48}$ could aid by identifying candidate regulators.

Recently, dCas12a (previously Cpf1), from Lachnospiraceae bacterium (dLbCas12a) or Acidaminococcus sp. (dAsCas12a), has become a popular alternative to dCas9 for gene regulation ${ }^{49,50}$. The Cas12a system has been popularized due to its ease of multiplexing; dCas12a uses smaller guide RNAs and is capable of processing these from a longer precursor CRISPR RNA ${ }^{51}$. Recent literature shows processing of 20 crRNA from a single precursor and simultaneous upregulation of 10 genes by dCas12a fused to a combination of the p65 activation domain together with the heat shock factor 1 in human embryonic kidney (HEK) 293 T cells, exemplifying 
the potential of multiplex gene regulation using dCas $12 \mathrm{a}^{52}$. A potential drawback for using dCas12a in fungi is the low activity at temperatures below $28{ }^{\circ} \mathrm{C}$, while most fungal species grow optimally at temperatures between 25 and $30^{\circ} \mathrm{C}$. However Roux and co-workers recently engineered an temperature tolerant Cas12a mutant (dLbCas12a ${ }^{\text {D156R }}$-VPR), which was successfully employed for CRISPRa mediated gene activation in A. nidulans at $25^{\circ} \mathrm{C}^{29}$. While dCas12a is an attractive choice when aiming to upregulate multiple genes simultaneously, for single target activation dCas9-VPR is still a good option. We got significant upregulation of an entire BGC using a single sgRNA targeting the TF of the BGC. For dLbCas12a based upregulation in A. nidulans (the unmutated dLbCas12a grown at $37^{\circ} \mathrm{C}$ ) multiple crRNAs were required for gene activation ${ }^{29}$. Another consideration when choosing a system is the different PAM requirement, NGG for (d)Cas9 and TTTN for (d)Cas12a. Depending on PAM availability in the genome one or the other could be preferable.

In conclusion we demonstrated that CRISPRa, specifically AMA1 vector-based expression of a dCas9-VPR fusion, can be used for the transcriptional activation of silent BGCs in P. rubens. We anticipate that the CRISPRa tool presented here can be widely used to awaken cryptic BGC in filamentous fungal species and thereby aid in the discovery of novel bioactive secondary metabolites.

\section{Methods}

Chemicals, reagents and oligodeoxyribonucleotides. All medium components and chemicals were purchased from Sigma-Aldrich (Zwijndrecht, Netherlands) or Merck (Darmstadt, Germany). Oligodeoxyribonucleotide primers (Supplementary Table S4) were obtained from Merck. Enzymes were obtained from Thermo Fisher Scientific (Waltham, MA) unless otherwise stated. For design of nucleic acid constructs, in-silico restriction cloning, Gibson Assembly and inspection of Sanger sequencing results, SnapGene (GSL Biotech) was used.

Vector construction. The Golden Gate technology based Modular Cloning (MoClo) system ${ }^{53}$ using Type IIS BpiI and BsaI restriction enzymes were employed for the construction of all vectors unless stated otherwise. Constructed vectors with their destination vectors and corresponding PCR fragments or DNA donor vectors can be found in Supplementary Table S5. PCR amplifications were conducted using KAPA HiFi HotStart ReadyMix (Roche Diagnostic, Switzerland) according to the instructions of the manufacturer. Internal BsaI, BpiI recognition sites were removed for MoClo compatibility.

dCas9-2xNLS-VPR was amplified from two different sources. NLS-VPR was amplified from pAG414GPDdCas9-VPR template (AddGene ID \# 63801) ${ }^{16}$ and dCas9-NLS was amplified with adding D10A, D839A, H840A and N863A modifications from synthetic spCas9 pYTK036 template, provided as part of the Yeast MoClo Toolkit (AddGene ID \# 65143) ${ }^{54}$. DNA sequence of the created dCas9-VPR fusion can be found in Supplementary Note S1a.

The HH and HDV ribozyme based "Plug-and-Play" sgRNA transcription unit was amplified in three parts, where the $g p d A$ promoter and the $\operatorname{tr} p C$ terminator together with the HDV self-cleaving sequence were amplified from vector pFC334 (AddGene ID \# 87846) ${ }^{33}$ and LacZ alpha gene was amplified from the MoClo ToolKit vector pICH41308 (AddGene ID \# 47998) ${ }^{53}$. The promoter of 40S ribosomal protein S8 of A. nidulans (AN0465.2, referred to as 40S) and the tif35 terminator of $P$. rubens Pc22g19890, as well as the transcription unit penDE-CPDsRed-SKL-TAct, were amplified from pVE2_10 (AddGene ID \#154228) ${ }^{36}$. The terbinafine selection marker as Pgpda-ergA-TamdS transcription unit was amplified from pCP1_45 $5^{41}$. The promoter of $p c b C(P c 21 g 21380$, IPNS) was amplified from pVE2_19 (AddGene ID \#154241) (36 $^{3}$ adding $80 \mathrm{bp}$ flanking regions for homologous recombination. The phleomycin selection marker was amplified from pDSM-JAK-10955 providing the P pcbCble-TCYC1 transcription unit, adding $80 \mathrm{bp}$ flanking regions for homologous recombination (Supplementary Fig. S1b, Supplementary Table S6).

Our autonomously replicating shuttle vector, carrying the AMA1 sequence, was based on pDSM-JAK-10955 where the Pgpda-DsRed-SKL-TpenDE transcriptional unit was removed using the BspTI and NotI restriction enzymes. The linear vector was treated with the Klenow Fragment and ligated to the circular vector using the T4 DNA Ligase according to the instructions of the manufacturer, creating a new AMA1 vector without DsRed expression. In order to create the CRISPRa vector, this vector was linearized with BspTI and was assembled by Gibson Cloning using PCR fragments G1, G2 G3 (Supplementary Table S5) carrying a terbinafine selection marker, dCas9-VPR and the sgRNA transcription unit respectively. CRISPRa vector pLM-AMA18.0 is deposited to AddGene under ID \#138,945. Parallel with this work a catalytically active spCas9 expressing vector was also established (pLM-AMA15.0 AddGene ID $\# 138,944$ ) and utilized for genome editing [manuscript in preparation].

sgRNA target design and cloning. Promoter sequences were analyzed with CCTop ${ }^{56}$ for possible CRISPR RNA guides with the following limitations: protospacer adjacent motif (PAM): NGG, target sequence length $20 \mathrm{bp}$, core length $12 \mathrm{bp}$, mismatches taken into account for prediction in core sequence 2, number of total mismatches 4 and using P. rubens Wisconsin $54-1255$ as the reference genome. Predicted protospacers were manually curated for minimizing off-target effects and selecting high CRISPRater ${ }^{57}$ scores. $^{2}$

Primers were designed to create 89 bp long dsDNA inserts by PCR, containing the unique 20 nt spacer sequence, the hammerhead ribozyme, the 6 bp inverted repeat of the $5^{\prime}$-end of the spacer sequence and the BsaI type II restriction enzyme recognition sites.

For cloning the inserts into the vector pAMA18.0 a modified MoClo protocol ${ }^{53}$ was used, using FastDigest BsaI (Thermo Fisher Scientific, Waltham, MA) restriction enzyme with an initial 10 min digestion, 50 cycles of digestion and ligation $\left(37^{\circ} \mathrm{C}\right.$ for $2 \mathrm{~min}, 16^{\circ} \mathrm{C}$ for $5 \mathrm{~min}$ ), followed by a final digestion step and a heat inactivation step. Correctly assembled vectors were identified with blue-white screening and confirmed by sequencing. After positive sequence verification and vector extraction, the created pAMA18.X (where X stands for the sgRNA 
ID) CRISPRa vector was introduced into the fungal strain of choice (DS68530_penDE-CP_DsRed or DS68530) creating the CRISPRa fungal strain AMA18.X (Fig. 1b, Supplementary Table S6).

Fungal strains and transformation. P. rubens strain DS68530 $0^{40}(\Delta$ penicillin-BGC, $\Delta h d f A$, derived from DS17690) was kindly provided by Centrient Pharmaceuticals (former DSM Sinochem Pharmaceuticals, Netherlands). Protoplasts of $P$. rubens were obtained $48 \mathrm{~h}$ post spore seeding in YGG medium and transformed using the methods and media as described previously ${ }^{14}$.

Mycelium was collected by centrifugation at $4000 \times \mathrm{g}$ for $8 \mathrm{~min}$ at $4{ }^{\circ} \mathrm{C}$. The pellet was resuspended in $50 \mathrm{ml} \mathrm{KC}$ solution $(60 \mathrm{~g} / \mathrm{l} \mathrm{KCl} ; 2 \mathrm{~g} / \mathrm{l}$ citric acid; $\mathrm{pH}$ set to 6.2$)$. After a second round of centrifugation, the pellet was resuspended in $18 \mathrm{ml} \mathrm{KC}$ solution and moved to sterile $100 \mathrm{ml}$ shake flask. The mycelium solution was supplemented with $25 \mathrm{mg} / \mathrm{ml}$ Glucanex Lysing Enzyme from Trichoderma harzianum (Sigma-Aldrich) and incubated at $25^{\circ} \mathrm{C}$ and 120 RPM for $90 \mathrm{~min}$. Successful protoplast formation was confirmed by microscopy. Protoplast solution was moved to a sterile falcon tube and was kept on ice when possible. Protoplast were diluted to $50 \mathrm{ml}$ using $\mathrm{KC}$ buffer and pelleted by centrifugation at $2770 \times \mathrm{g}$ for $5 \mathrm{~min}$ at $4{ }^{\circ} \mathrm{C}$ (same settings were used in all subsequent centrifugation steps). Protoplast pellets were resuspended in $25 \mathrm{ml} \mathrm{KC}$ buffer followed by addition of $25 \mathrm{ml}$ STC buffer (219 g/l sorbitol, $5.5 \mathrm{~g} / \mathrm{l} \mathrm{CaCl}_{2}, 10 \mathrm{mM}$ Tris- $\mathrm{HCl} \mathrm{pH} \mathrm{7.5;} \mathrm{pH}$ set to 7.5 8.0). After centrifugation, pelleted protoplasts were resuspended in $50 \mathrm{ml}$ STC and counted by microscopy using a counting chamber. After centrifugation protoplasts were resuspended in STC to obtain $2 \times 10^{7}$ protoplasts $/ \mathrm{ml}$ (approximately $1-5 \mathrm{ml}$ ). These protoplasts were used fresh, or stored at $-80^{\circ} \mathrm{C}$ in $10 \%(\mathrm{w} / \mathrm{v})$ PVP-40 (Polyvinylpyrrolidone, Sigma-Aldrich) dissolved in STC as a cryopreservation buffer.

Protoplasts were transformed using PEG-mediated transformation ${ }^{14}$. In short, $200 \mu$ protoplast solution $\left(\sim 2 \times 10^{7}\right.$ protoplasts $\left./ \mathrm{ml}\right)$ was added to a sterile $12-\mathrm{ml}$ Greiner tube on ice, and were mixed with 1-8 $\mu$ g DNA (in maximum $50 \mu \mathrm{l}$ ) and $200 \mu \mathrm{l} 20 \%$ PEG-4000 solution (33 ml 60\% PEG-4000; $67 \mathrm{ml}$ STC buffer; $109.5 \mathrm{~g}$ sorbitol; $5 \mathrm{ml} 1 \mathrm{M}$ TRIS-HCl butter $\mathrm{pH} 7.5$; in final volume of $250 \mathrm{ml}$ ). Protoplasts were incubated on ice for $30 \mathrm{~min}$. Tubes were supplemented with $1.5 \mathrm{ml} 60 \%$ PEG-4000 solution (60 g PEG-4000 dissolved in $40 \mathrm{ml} \mathrm{H}_{2} \mathrm{O}$

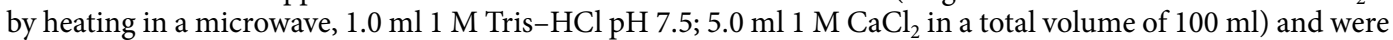
homogenized completely by rotating the tube for $2 \mathrm{~min}$. The tubes were placed in a $25^{\circ} \mathrm{C}$ incubator for $25 \mathrm{~min}$. $1.2 \mathrm{M}$ sorbitol was added to a total of $11 \mathrm{ml}$, and protoplasts were pelleted by centrifugation at $2770 \times \mathrm{g}$ for $5 \mathrm{~min}$ at $25^{\circ} \mathrm{C}$. Protoplasts were carefully resuspended in $1 \mathrm{ml} 1.2 \mathrm{M}$ sorbitol and 100, 200 and $300 \mathrm{ul}$ was plated on solid transformation medium.

When transforming the CRISPRa AMA1 vectors, total DNA did not exceed $1 \mu \mathrm{g}$. For Cas9-mediated genome editing of macR:OE and penDE-CP_DsRed strains, the appropriate Cas9 RNP mixtures were added. Using CRISPR/Cas9 ribonucleoprotein (RNP) facilitated co-transformation ${ }^{13,36}$, marker free DNA was delivered in 10:1 molar ratio compared to the fungal marker, not exceeding $8 \mu \mathrm{g}$ total DNA. Synthetized sgRNAs were prepared using MEGAscript T7 Transcription Kit (Thermo Fisher Scientific, Waltham, MA) from PCR generated DNA templates, and the Cas9 protein was overexpressed in E. coli T7 Express lys $Y$ from pET28a/Cas9-Cys (AddGene ID \#53,261). For each transformation, separate Cas9 RNP mixtures were formed by mixing $27 \mu \mathrm{g}$ Cas 9 protein (up to $15 \mu \mathrm{l}$ ), $4 \mu \mathrm{l}$ of synthetized sgRNA; $35 \mu \mathrm{l} 2 \times \mathrm{STC}$ and $30 \mu \mathrm{l} 10 \times$ Cas 9 activity buffer (HEPES $4.76 \mathrm{~g} / \mathrm{l} ; \mathrm{KCl}$ $11.18 \mathrm{~g} / \mathrm{l}$; EDTA $0.029 \mathrm{~g} / \mathrm{l} ; \mathrm{MgCl}_{2} \times 7 \mathrm{H}_{2} \mathrm{O} 2.03 \mathrm{~g} / \mathrm{l}$; $\mathrm{pH}$ set to 7.5 ; DTT $0.08 \mathrm{~g} / \mathrm{l}$ ).

Media and culture conditions. Solid transformation medium was prepared using SAG solid medium (Sucrose $375 \mathrm{~g} / \mathrm{l}$ : Agar $15 \mathrm{~g} / \mathrm{l}$; Glucose Monohydrate $10 \mathrm{~g} / \mathrm{l}$ ) supplemented, in this order, with $4 \mathrm{ml} / \mathrm{l}$ Trace Element Solution ${ }^{58}$, $25.7 \mathrm{ml} / \mathrm{l}$ stock solution $\mathrm{A} ; 25.7 \mathrm{ml} / \mathrm{l}$ stock solution B and $2.4 \mathrm{ml} / \mathrm{l} 4 \mathrm{M} \mathrm{KOH}$ (where stock solution A contained the following: $\mathrm{KCl} 28.80 \mathrm{~g} / \mathrm{l} ; \mathrm{KH}_{2} \mathrm{PO}_{4} 60.8 \mathrm{~g} / \mathrm{l} ; \mathrm{NaNO}_{3} 240 \mathrm{~g} / \mathrm{l}$, at pH 5.5 (adjusted using $\mathrm{KOH}$ ) and stock solution B contained: $\mathrm{MgSO} 4 \cdot 7 \mathrm{H} 2 \mathrm{O}$ at $20.80 \mathrm{~g} / \mathrm{l}$ ). Selection for the terbinafine marker based macR:OE cassette and all CRISPRa vector carrying transformants was carried out using $1.1 \mu \mathrm{g} / \mathrm{ml}$ terbinafine hydrochloride (Sigma-Aldrich) in the solid transformation medium. Terbinafine was supplemented in all media of consecutive experiments, whereas selection for penDE-CP_DsRed and PpcbC-ble-tCYC1 co-transformation was done using medium containing $50 \mu \mathrm{g} / \mathrm{ml}$ phleomycin (Invivogen, San Diego, CA). For each strain, 2 separate transformant colonies were selected as replicates and re-streaked individually on solid R-agar (see below) medium and cultivated for 7 days on $25^{\circ} \mathrm{C}$ to produce spores, which were immobilized on lyophilized rice grains and used for further experiments. Schematic representation of engineering DS68530_penDE-CP_DsRed and macR:OE control strains, using CRISPR/Cas9 mediated homologous recombination-based co-transformation into DS68530, is shown on Supplementary Figure S1. For each created strain, transformed DNA is listed in Supplementary Table S6.

For shake-flask liquid cultures, spores immobilized on lyophilized rice grains $\left(0.2 \times 10^{6}-2 \times 10^{6} \mathrm{spores} / \mathrm{ml}\right)$ were precultured for $24 \mathrm{~h}$ in YGG medium ${ }^{59}$ before inoculation (1:7.5) into $30 \mathrm{ml}$ Secondary Metabolite Producing (SMP) medium ${ }^{59}$ (Penicillin Producing Medium-PPM-without supplemented phenoxyacetic acid or phenylacetic acid), supplemented with $1.1 \mu \mathrm{g} / \mathrm{ml}$ terbinafine. Cultures were grown at $25^{\circ} \mathrm{C}$ in a rotary incubator at 200 RPM for 5 days, after which mycelium was collected for total RNA extraction as well as extraction of secondary metabolites by vacuum filtration over filter paper. Solid R-agar medium ${ }^{58}$ was used for sporulation, SMP-agar (SMP medium supplemented with $15 \mathrm{~g} / \mathrm{l}$ agar-agar) was used for cultivation, and for secondary metabolite extraction. All solid agar cultures were incubated at $25^{\circ} \mathrm{C}$.

Total RNA extraction and cDNA synthesis. Total RNA was extracted from mycelium collected from cultures grown in SMP for 5 days at $25^{\circ} \mathrm{C}$. Wet biomass $(\sim 200 \mathrm{mg})$ was added to a screw cap tube containing $900 \mu \mathrm{l}$ Trizol reagent (Thermo Fisher Scientific, Waltham, MA), $125 \mu$ l chloroform and glass beads ( 0.75-1 mm, 500-600 mg). The samples were stored at $-80^{\circ} \mathrm{C}$ until RNA isolation. The mycelium was disrupted 
using the FastPrep FP120 system (Qbiogene, Carlsbad, CA), followed by total RNA isolation using the phenolchloroform extraction method. In short, after cell disruption phases were separated by centrifugation (10 min at $14,000 \times g$, the upper phase was transferred to a new tube, followed by a chloroform extraction step (phase separation: $5 \mathrm{~min}$ at $12,000 \times \mathrm{g}$ ). RNA was precipitated by the addition of 1 volume isopropanol and incubated on ice for at least $10 \mathrm{~min}$, followed by centrifugation $(10 \mathrm{~min}$ at $12,000 \times \mathrm{g})$. Finally, the RNA was resuspended in milliQ $\mathrm{H}_{2} \mathrm{O}$. DNAse treatment was done using the TURBO DNA-free Kit (Thermo Fisher Scientific, Waltham, MA), and the RNA concentration was determined using Nanodrop. cDNA was synthesized using RevertAid $\mathrm{H}$ Minus First Strand cDNA synthesis kit (Thermo Fisher Scientific, Waltham, MA) according to the manufacturer's instructions for highly structured mRNAs using oligo $(\mathrm{dT})_{18}$ primers and $1 \mu \mathrm{g}$ of total RNA as template in a $20 \mu$ reaction.

qPCR analysis. Primers used to analyze expression DsRed, macR, macA and macJ can be found in Supplementary Table S7. Primers were, when possible, designed to overlap an intron-exon junction to avoid amplification on gDNA. The $\gamma$-actin gene (Pc20g11630) was used as a control for normalization. The $25 \mu \mathrm{l}$ qCR reaction contained $4 \mu \mathrm{l}$ of a $20 \times$ diluted cDNA synthesis reaction, $0.6 \mu \mathrm{M}$ each of forward and reverse primer, and $12.5 \mu \mathrm{l}$ SensiMix SYBR Hi-ROX master mix (Meridian Bioscience, Memphis, TN). Expression levels were determined with a MiniOpticon system (Bio-Rad, Hercules, CA) using the Bio-Rad CFX manager software, the threshold cycle $(C t)$ values were determined automatically by regression. Thermocycler conditions were as follows: $95^{\circ} \mathrm{C}$ for $10 \mathrm{~min}$, followed by 40 cycles of $95^{\circ} \mathrm{C}$ for $15 \mathrm{~s}, 60^{\circ} \mathrm{C}$ for $30 \mathrm{~s}$, and $72^{\circ} \mathrm{C}$ for $30 \mathrm{~s}$. Thereafter, a melting curve was generated to determine the specificity of the qPCRs.

LC-MS sample preparation. For secondary metabolite analysis samples were taken from vacuum filtered mycelium or from solid SMP-agar medium as $3 \times 1 \mathrm{~cm}$ diameter plugs. The plugs were transferred to a $4.0 \mathrm{ml}$ glass vial and $1 \mathrm{ml}$ acetone supplemented with $4 \mu \mathrm{n}$-Dodecyl- $\beta$-D-maltoside (DDM) $(10 \mathrm{mg} / \mathrm{ml}$ in methanol) was added as internal standard. The plugs were extracted ultrasonically for $60 \mathrm{~min}$, after which the extracts were transferred to a clean vial and dried under a nitrogen stream at $25^{\circ} \mathrm{C}$. Dried extracts were resuspended in $200 \mu \mathrm{l}$ methanol:milliQ- $\mathrm{H}_{2} \mathrm{O}(1: 1)$ and filtered via a $0.2 \mu \mathrm{m}$ PTFE syringe filter before the used for LC-MS analysis.

LC-MS metabolite analysis. Metabolite analysis was performed using an Accella1250 UHPLC system coupled to a benchtop ESI-MS Orbitrap Exactive (Thermo Fisher Scientific, Waltham, MA) mass-spectrometer. A sample of $5 \mu \mathrm{l}$ was injected onto a Waters Acquity CSH C18 UPLC (UHPLC) column $(150 \times 2.1 \mathrm{~mm}, 1.7 \mu \mathrm{m}$ particle size) operating at $40{ }^{\circ} \mathrm{C}$ with a flow rate of $300 \mu \mathrm{l} / \mathrm{min}$. Separation of the compounds was achieved by using a water-acetonitrile gradient system starting from $90 \%$ of solvent A (milliQ-water) and $5 \%$ solvent B ( $100 \%$ acetonitrile). $5 \%$ of solvent C ( $2 \%$ formic acid) was continuously added to maintain a final concentration of $0.1 \%$ of formic acid in the mobile phase. After $5 \mathrm{~min}$ of initial isocratic flow, the first linear gradient reached $60 \%$ of B at $30 \mathrm{~min}$, and the second $95 \%$ of B at $35 \mathrm{~min}$. A purge step for $10 \mathrm{~min}$ at $90 \%$ of B was followed by column equilibration for $15 \mathrm{~min}$ at the initial conditions. The column eluent was directed to a HESI-II ion source attached to the Exactive Orbitrap mass spectrometer operating at the scan range (m/z 80-1600 Da) and alternating between positive/negative polarity modes for each scan. LC-MS data were analyzed using the Thermo Scientific Xcalibur 2.2 processing software by applying the Genesis algorithm for peak detection and manual integration on the sum of the whole spectra of selected ions. The extracted ion counts of investigated compounds were normalized to the DDM internal standard and represented relative to the average detected values from the MacR:OE strain replicates. In addition to LC-MS only UV-VIS absorption was monitored at 220,354 and $700 \mathrm{~nm}$. Ions corresponding to the $[\mathrm{M}+\mathrm{H}]^{+}$pseudo molecular ions of the final steps of the macrophorin biosynthesis pathway (Macrophorin A, macrophorin D and 4'-oxomacrophorin D) were identified in chromatographic peaks (1), (2) and (3) respectively and were selected for further analysis. The peaks recorded by each channel for (1), (2) and (3) in match in retention time. The chromatogram recorded at $700 \mathrm{~nm}$ showed the best signal-to-noise ratio. (Fig. 3f, Supplementary Fig. S4). Due to the necessity of adding an in-line UV-VIS detector between the MS and the column to generate UV-VIS chromatograms, small discrepancy in Rt between different datasets was observed.

Biolector. Spores (immobilized on 20 rice grains) were used to inoculate $10 \mathrm{ml}$ SMP and cultures were incubated for $48 \mathrm{~h}$ in a rotary incubator at $200 \mathrm{rpm}$ and $25^{\circ} \mathrm{C}$. For BioLector analysis and analysis of growth in FlowerPlate (MTP-48-B) wells, this pre-grown mycelium was diluted 8 times in fresh SMP, supplemented with $1.1 \mu \mathrm{g} / \mathrm{ml}$ terbinafine (except for parent strain DS68530). The $1 \mathrm{ml}$ cultures were grown in the BioLector microbioreactor system (M2Plabs, Baesweiler, Germany), shaking at $800 \mathrm{rpm}$ at $25^{\circ} \mathrm{C}$. In the BioLector, biomass was measured via scattered light at $620 \mathrm{~nm}$ excitation without an emission filter. The fluorescence of DsRed-SKL was measured every $30 \mathrm{~min}$ with "DsRed I" $550 \mathrm{~nm}$ (bandpass: $10 \mathrm{~nm}$ ) excitation filter and $580 \mathrm{~nm}$ (bandpass: $10 \mathrm{~nm}$ ) emission filter. Data were obtained from 3 separate experiments, each consisting of 2-3 biological replicates. The data obtained from the BioLector experiments were analyzed and presented using the TIBCO Spotfire Software (TIBCO Software Inc., Palo Alto, CA).

Fluorescence microscopy. For visualization of DsRed-SKL fluorescent protein, liquid shake-flask cultures were cultivated for 5 days in SMP, and mycelium was collected and re-suspended in phosphate-buffered saline $\left(58 \mathrm{mM} \mathrm{Na}_{2} \mathrm{HPO}_{4} ; 17 \mathrm{mM} \mathrm{NaH}_{2} \mathrm{PO}_{4} ; 68 \mathrm{mM} \mathrm{NaCl}, \mathrm{pH}\right.$ 7.3). Confocal imaging was performed on a Carl Zeiss LSM800 confocal microscope using $20 \times$ objective and ZEN 2009 software (Carl Zeiss, Oberkochen, Germany). The DsRed signal was visualized by excitation with a $543 \mathrm{~nm}$ helium neon laser (Lasos Lasertechnik, Jena, Germany), and emission was detected using a 565 to $615 \mathrm{~nm}$ band-pass emission filter ${ }^{60}$. 
Bio-assay. Macrophorin producing strains were tested for antimicrobial activity against Micrococcus luteus as follows: Supernatant of $P$. rubens strains carrying either the pAMA18.0, pAMA18.4, pAMA18.5 vector and the macR:OE strain was collected after 5 days of growth in liquid SMP medium and concentrated $10 \times$ in an Eppendorf Concentrator Plus $\left(30^{\circ} \mathrm{C}\right.$, vacuum for aqua solutions setting). An overlay of soft LA-agar (1\%) inoculated with $M$. luteus to an OD600 of 0.125 was poured on top of an agar (1\%) bottom layer with Oxford Towers $(8 \times 10 \mathrm{~mm})$ spaced out evenly. The Oxford Towers were removed aseptically and $100 \mu \mathrm{l}$ of the $10 \times$ concentrated supernatant was loaded in the resulting wells as indicated. The plate was incubated at $30^{\circ} \mathrm{C}$ for $24 \mathrm{~h}$ before imaging. The experiment was performed in triplicate.

Received: 13 October 2020; Accepted: 21 December 2020

Published online: 13 January 2021

\section{References}

1. Keller, N. P. Fungal secondary metabolism: Regulation, function and drug discovery. Nat. Rev. Microbiol. 17, 167-180 (2019).

2. Kück, U., Bloemendal, S. \& Teichert, I. Putting fungi to work: Harvesting a cornucopia of drugs, toxins, and antibiotics. PLoS Pathog. 10, 3-6 (2014).

3. Schueffler, A. \& Anke, T. Fungal natural products in research and development. Nat. Prod. Rep. 31, 1425-1448 (2014).

4. Scharf, D. H., Heinekamp, T. \& Brakhage, A. A. Human and plant fungal pathogens: The role of secondary metabolites. PLoS Pathog. 10, 10-12 (2014).

5. Medema, M. H. et al. antiSMASH: Rapid identification, annotation and analysis of secondary metabolite biosynthesis gene clusters in bacterial and fungal genome sequences. Nucleic Acids Res. 39, W339-W346 (2011).

6. Kautsar, S. A. et al. MIBiG 2.0: A repository for biosynthetic gene clusters of known function. Nucleic Acids Res. 48, D454-D458 (2019).

7. Brakhage, A. A. \& Schroeckh, V. Fungal secondary metabolites-strategies to activate silent gene clusters. Fungal Genet. Biol. 48, $15-22(2011)$.

8. Lim, F. Y., Sanchez, J. F., Wang, C. C. C. \& Keller, N. P. Toward awakening cryptic secondary metabolite gene clusters in filamentous fungi. Meth. Enzymol. 517, 303-324 (2012).

9. Knott, G. J. \& Doudna, J. A. CRISPR-Cas guides the future of genetic engineering. Science 361, 866-869 (2018).

10. Pickar-Oliver, A. \& Gersbach, C. A. The next generation of CRISPR-Cas technologies and applications. Nat. Rev. Mol. Cell Biol. 20, 490-507 (2019).

11. Schuster, M. \& Kahmann, R. CRISPR-Cas9 genome editing approaches in filamentous fungi and oomycetes. Fungal Genet. Biol. 130, 43-53 (2019).

12. Song, R. et al. CRISPR/Cas9 genome editing technology in filamentous fungi: Progress and perspective. Appl. Microbiol. Biotechnol. 103, 6919-6932 (2019).

13. Pohl, C., Kiel, J. A. K. W., Driessen, A. J. M., Bovenberg, R. A. L. \& Nygård, Y. CRISPR/Cas9 based genome editing of Penicillium chrysogenum. ACS Synth. Biol. 5, 754-764 (2016).

14. Pohl, C., Mózsik, L., Driessen, A. J. M., Bovenberg, R. A. L. \& Nygård, Y. I.Genome editing in Penicillium chrysogenum using Cas9 ribonucleoprotein particles. In Synthetic Biology Methods in Molecular Biology Vol. 1772 (ed. Braman, J.) 213-232 (Humana Press Inc., Heidelberg, 2018).

15. Houbraken, J., Frisvad, J. C. \& Samson, R. A. Fleming's penicillin producing strain is not Penicillium chrysogenum but P. rubens. IMA Fungus 2, 87-95 (2011).

16. Chavez, A. et al. Highly efficient Cas9-mediated transcriptional programming. Nat. Methods 12, 326-328 (2015).

17. Gilbert, L. A. et al. CRISPR-mediated modular RNA-guided regulation of transcription in eukaryotes. Cell 154, 442-451 (2013).

18. Qi, L. S. et al. Repurposing CRISPR as an RNA-suided platform for sequence-specific control of gene expression. Cell 152, 1173-1183 (2013).

19. Hilton, I. B. et al. Epigenome editing by a CRISPR-Cas9-based acetyltransferase activates genes from promoters and enhancers. Nat. Biotechnol. 33, 510-517 (2015).

20. Vojta, A. et al. Repurposing the CRISPR-Cas9 system for targeted DNA methylation. Nucleic Acids Res. 44, 5615-5628 (2016).

21. Chen, B. et al. Dynamic imaging of genomic loci in living human cells by an optimized CRISPR/Cas system. Cell 155, 1479-1491 (2013).

22. Gaudelli, N. M. et al. Programmable base editing of T to G C in genomic DNA without DNA cleavage. Nature 551, 464-471 (2017).

23. Brezgin, S., Kostyusheva, A., Kostyushev, D. \& Chulanov, V. Dead cas systems: Types, principles, and applications. Int. J. Mol. Sci. 20, 1-26 (2019).

24. Xu, X. \& Qi, L. S. A CRISPR-dCas toolbox for genetic engineering and synthetic biology. J. Mol. Biol. 431, 34-47 (2019).

25. Deaner, M. \& Alper, H. S. Systematic testing of enzyme perturbation sensitivities via graded dCas 9 modulation in Saccharomyces cerevisiae. Metab. Eng. 40, 14-22 (2017).

26. Cámara, E., Lenitz, I. \& Nygård, Y. A CRISPR activation and interference toolkit for industrial Saccharomyces cerevisiae strain KE6-12. Sci. Rep. 10, 14605 (2020).

27. Schwartz, C., Curtis, N., Löbs, A.-K. \& Wheeldon, I. Multiplexed CRISPR activation of cryptic sugar metabolism enables Yarrowia Lipolytica growth on cellobiose. Biotechnol. J. 13, 1700584 (2018).

28. Román, E., Coman, I., Prieto, D., Alonso-Monge, R. \& Pla, J. Implementation of a CRISPR-based system for gene regulation in Candida albicans. mSphere 4, e00001-19 (2019).

29. Roux, I. et al. CRISPR-mediated activation of biosynthetic gene clusters for bioactive molecule discovery in filamentous fungi. ACS Synth. Biol. 9, 1843-1854 (2020).

30. Fierro, F., Kosalková, K., Gutiérrez, S. \& Martín, J. F. Autonomously replicating plasmids carrying the AMA1 region in Penicillium chrysogenum. Curr. Genet. 29, 482-489 (1996).

31. Aleksenko, A. \& Clutterbuck, A. J. Autonomous plasmid replication in Aspergillus nidulans AMA1 and MATE elements. Fungal Genet. Biol. 21, 373-387 (1997).

32. Wang, Q. \& Coleman, J. J. Progress and challenges: Development and implementation of CRISPR/Cas9 technology in filamentous fungi. Comput. Struct. Biotechnol. J. 17, 761-769 (2019).

33. Nødvig, C. S., Nielsen, J. B., Kogle, M. E. \& Mortensen, U. H. A CRISPR-Cas9 system for genetic engineering of filamentous fungi. PLoS One 10, e0133085 (2015).

34. Gao, Y. \& Zhao, Y. Self-processing of ribozyme-flanked RNAs into guide RNAs in vitro and in vivo for CRISPR-mediated genome editing. J. Integr. Plant Biol. 56, 343-349 (2014). 
35. Engler, C., Gruetzner, R., Kandzia, R. \& Marillonnet, S. Golden gate shuffling: A one-pot DNA shuffling method based on type ils restriction enzymes. PLoS One 4, e5553 (2009).

36. Mózsik, L., Büttel, Z., Bovenberg, R. A. L., Driessen, A. J. M. \& Nygård, Y. Synthetic control devices for gene regulation in Penicillium chrysogenum. Microb. Cell Fact. 18, 203 (2019).

37. Holm, D. K. et al. Molecular and chemical characterization of the biosynthesis of the 6-MSA-derived meroterpenoid yanuthone D in Aspergillus niger. Chem. Biol. 21, 519-529 (2014).

38. Petersen, L. M. et al. Characterization of four new antifungal yanuthones from Aspergillus niger. J. Antibiot. (Tokyo) 68, 201-205 (2015).

39. Tang, M. C. et al. Late-stage terpene cyclization by an integral membrane cyclase in the biosynthesis of isoprenoid epoxycyclohexenone natural products. Org. Lett. 19, 5376-5379 (2017).

40. Salo, O. V. et al. Genomic mutational analysis of the impact of the classical strain improvement program on $\beta$-lactam producing Penicillium chrysogenum. BMC Genomics 16, 937 (2015).

41. Pohl, C. et al. A Penicillium rubens platform strain for secondary metabolite production. Sci. Rep. 10, 7630 (2020).

42. Bugni, T. S. et al. Yanuthones: Novel metabolites from a marine isolate of Aspergillus niger. J. Org. Chem. 65, 7195-7200 (2000).

43. Nielsen, J. C. et al. Global analysis of biosynthetic gene clusters reveals vast potential of secondary metabolite production in Penicillium species. Nat. Microbiol. 2, 17044 (2017).

44. Kuivanen, J., Korja, V., Holmström, S. \& Richard, P. Development of microtiter plate scale CRISPR/Cas9 transformation method for Aspergillus niger based on in vitro assembled ribonucleoprotein complexes. Fungal Biol. Biotechnol. 6, 3 (2019).

45. Van Leeuwe, T. M. et al. Efficient marker free CRISPR/Cas9 genome editing for functional analysis of gene families in filamentous fungi. Fungal Biol. Biotechnol. 6, 13 (2019).

46. Farzadfard, F., Perli, S. D. \& Lu, T. K. Tunable and multifunctional eukaryotic transcription factors based on CRISPR/Cas. ACS Synth. Biol. 2, 604-613 (2013).

47. Blin, K. et al. AntiSMASH 5.0: Updates to the secondary metabolite genome mining pipeline. Nucleic Acids Res. 47, W81-W87 (2019).

48. Wolf, T., Shelest, V., Nath, N. \& Shelest, E. CASSIS and SMIPS: Promoter-based prediction of secondary metabolite gene clusters in eukaryotic genomes. Bioinformatics 32, 1138-1143 (2016).

49. Tak, Y. E. et al. Inducible and multiplex gene regulation using CRISPR-Cpf1-based transcription factors. Nat. Methods 14, 11631166 (2017).

50. Liu, Y. et al. Engineering cell signaling using tunable CRISPR-Cpf1-based transcription factors. Nat. Commun. 8, 1-8 (2017),

51. Fonfara, I., Richter, H., Bratovič, M., Le Rhun, A. \& Charpentier, E. The CRISPR-associated DNA-cleaving enzyme Cpf1 also processes precursor CRISPR RNA. Nature 532, 517-521 (2016).

52. Campa, C. C., Weisbach, N. R., Santinha, A. J., Incarnato, D. \& Platt, R. J. Multiplexed genome engineering by Cas12a and CRISPR arrays encoded on single transcripts. Nat. Methods 16, 887-893 (2019).

53. Weber, E., Engler, C., Gruetzner, R., Werner, S. \& Marillonnet, S. A modular cloning system for standardized assembly of multigene constructs. PLoS ONE 6, e16765 (2011).

54. Lee, M. E., DeLoache, W. C., Cervantes, B. \& Dueber, J. E. A highly characterized yeast toolkit for modular multipart assembly. ACS Synth. Biol. 4, 975-986 (2015).

55. Bovenberg R.A.L., Kiel, J. A. K. W., Wenzel, T. J., Los, A. P. Vector-Host system. World Intellect. Prop. Organ. Application number $\mathrm{WO} / 2012 / 123429(2012)$.

56. Stemmer, M., Thumberger, T., del Sol Keyer, M., Wittbrodt, J. \& Mateo, J. L. CCTop: An intuitive, flexible and reliable CRISPR/ Cas9 target prediction tool. PLoS One 10, e0124633 (2015).

57. Labuhn, M. et al. Refined sgRNA efficacy prediction improves large- and small-scale CRISPR-Cas9 applications. Nucleic Acids Res. 46, 1375-1385 (2017).

58. Kovalchuk, A., Weber, S. S., Nijland, J. G., Bovenberg, R. A. L. \& Driessen, A. J. M. Fungal ABC transporter deletion and localization analysis. Methods Mol. Biol. 835, 1-16 (2012).

59. Weber, S. S., Polli, F., Boer, R., Bovenberg, R. A. L. \& Driessen, A. J. M. Increased penicillin production in Penicillium chrysogenum production strains via balanced overexpression of isopenicillin n acyltransferase. Appl. Environ. Microbiol. 78, 7107-7113 (2012).

60. Singh, R. et al. Hansenula polymorpha Pex37 is a peroxisomal membrane protein required for organelle fission and segregation. FEBS J. 287, 1742-1757 (2020).

\section{Acknowledgements}

The authors would like to thank Arjen M. Krikken for the assistance with confocal fluorescence microscopy imaging.

\section{Author contributions}

L.M. and M.H. contributed equally to this work. LC-MS analysis was carried out by N.A.W.K and L.M. BioLector analysis was carried out by Y.N.. L.M. designed the experiments. L.M. and M.H. carried out all other experiments and wrote the manuscript with critical feedback and help from N.A.W.K., R.A.L.B., Y.N., and A.J.M.D.. Y.N., R.A.L.B., and A.J.M.D. conceived the original idea.

\section{Funding}

The project leading to this application has received funding from the European Union's Horizon 2020 Research and Innovation Programme under the Marie Skłodowska-Curie grant agreement No. 713482 (ALERT Program). M.H. received funding from the Swedish Research Council [2019-00596]. N.A.W.K. was supported by the Building Blocks of Life programme, which is subsidized by the Netherlands Organization for Scientific Research (NWO) [BBOL.16.010].

\section{Competing interests}

The authors declare no competing interests.

\section{Additional information}

Supplementary Information The online version contains supplementary material available at https://doi.org/ 10.1038/s41598-020-80864-3.

Correspondence and requests for materials should be addressed to A.J.M.D.

Reprints and permissions information is available at www.nature.com/reprints. 
Publisher's note Springer Nature remains neutral with regard to jurisdictional claims in published maps and institutional affiliations.

(c) (i) Open Access This article is licensed under a Creative Commons Attribution 4.0 International License, which permits use, sharing, adaptation, distribution and reproduction in any medium or format, as long as you give appropriate credit to the original author(s) and the source, provide a link to the Creative Commons licence, and indicate if changes were made. The images or other third party material in this article are included in the article's Creative Commons licence, unless indicated otherwise in a credit line to the material. If material is not included in the article's Creative Commons licence and your intended use is not permitted by statutory regulation or exceeds the permitted use, you will need to obtain permission directly from the copyright holder. To view a copy of this licence, visit http://creativecommons.org/licenses/by/4.0/.

(C) The Author(s) 2021, corrected publication 2021 